\title{
De novo assembly of potential linear artificial chromosome constructs capped with expansive telomeric repeats
}

\author{
Li Lin, Dal-Hoe Koo, Wenli Zhang, Joseph St Peter and Jiming Jiang*
}

\begin{abstract}
Background: Artificial chromosomes (ACs) are a promising next-generation vector for genetic engineering. The most common methods for developing AC constructs are to clone and combine centromeric DNA and telomeric DNA fragments into a single large DNA construct. The AC constructs developed from such methods will contain very short telomeric DNA fragments because telomeric repeats can not be stably maintained in Escherichia coli.

Results: We report a novel approach to assemble AC constructs that are capped with long telomeric DNA. We designed a plasmid vector that can be combined with a bacterial artificial chromosome (BAC) clone containing centromeric DNA sequences from a target plant species. The recombined clone can be used as the centromeric DNA backbone of the AC constructs. We also developed two plasmid vectors containing short arrays of plant telomeric DNA. These vectors can be used to generate expanded arrays of telomeric DNA up to several kilobases. The centromeric DNA backbone can be ligated with the telomeric DNA fragments to generate AC constructs consisting of a large centromeric DNA fragment capped with expansive telomeric DNA at both ends.

Conclusions: We successfully developed a procedure that circumvents the problem of cloning and maintaining long arrays of telomeric DNA sequences that are not stable in E. coli. Our procedure allows development of AC constructs in different eukaryotic species that are capped with long and designed sizes of telomeric DNA fragments.
\end{abstract}

\begin{abstract}
Introduction
Artificial chromosomes (ACs) were first developed in budding yeast Saccharomyces cerevisiae through the cloning and assembling of three DNA elements: the centromere, telomeres and origins of replication [1]. The success of yeast artificial chromosomes (YACs) was a driving force for the development of artificial chromosomes in multicellular eukaryotes. Human artificial chromosomes (HACs) and plant artificial chromosomes (PACs) can not only provide important tools for studying chromosome structure and function, but also hold great potential as next generation vectors for human gene therapy and plant genetic engineering [2-4]. Development of both HACs and PACs have been reported after a decade long effort involving many laboratories [5-9].
\end{abstract}

\footnotetext{
* Correspondence: jjiang1@wisc.edu

* Correspondence: jjiang1@wisc.edu 53706, USA
}

Several different techniques have been developed to assemble AC constructs in mammalian and plant species. Most of these techniques have focused on combining centromeric DNA with telomeric DNA fragments. Origins of replication are poorly defined in higher eukaryotes but presumably exist throughout their genomes [10]. Thus, the centromeric and telomeric DNA used in AC constructs may contain sequence motives required for DNA replication. The most common approach for developing artificial chromosomes is using a cloned centromeric DNA fragment as the backbone of the constructs. YACs or bacterial artificial chromosomes (BACs) containing centromeric DNA were commonly used in construct development [2]. Subsequently, telomeric DNA fragments are added to the ends of the YAC or BAC insert $[6,7,9]$. This results in a DNA molecule containing a large centromeric DNA fragment capped with telomeric DNA from the targeted animal or plant species. 
One of the main shortfalls in the current approaches of HAC and PAC assembly is the very short telomeric DNA fragments included in the constructs. Satellite repeats, including telomeric repeats, cannot be stably maintained in E. coli. Thus, if the HAC/PAC construct or part of the construct containing the telomeric DNA fragments is propagated in $E$. coli, any long arrays of the telomeric DNA may be partially or significantly deleted or rearranged. Due to this problem the lengths of the telomeric DNA of previously reported HAC/PAC constructs were all shorter than those of the native chromosomes, which can reduce the efficiency of artificial chromosome formation and affect the stability of resulting minichromosomes $[11,12]$.

We sought a new strategy to circumvent this problem. Here we report the development of two telomeric DNA vectors that can be used to generate long telomeric DNA fragments up to several kilobases. We also developed a vector that can be combined with BAC clones containing large centromeric DNA inserts. The cloned centromeric DNA can be subsequently recombined with expansive telomeric DNA resulting in an in vitro system for the production of AC constructs. This AC assembly system allows the generation of AC constructs capped with telomeric DNA in different sizes. The technique can be applied in different plant as well as animal species.

\section{Results}

Development of a vector as a centromeric DNA backbone We first developed the pLL-EH vector (Figure 1A). This vector $(12,012 \mathrm{bp})$ consists of two DNA fragments. The first fragment $(6,212$-bp) was isolated from the BAC pBeloBAC11 [13] by double digestion with PciI and SalI (Figure 1A). This fragment contains all the genes required for stable propagation and maintenance of large DNA fragments in E. coli. The second fragment was synthesized containing a number of restriction sites for cloning and recombination. A hygromycin resistance gene $(H p t)$ and a reporter gene Egfp were also inserted into this fragment (Figure 1A). The attP1 site can be used for in vitro site-specific recombination with the attB1 site from telomeric DNA vectors. The lox71 and the $\phi$ C31 attB1 sites can be used to insert additional DNA sequences into the vector or future potential artificial chromosomes of transgenic plants.

A BAC clone containing centromeric DNA can be ligated with pLL-EH to form a pLL-EHC vector (Figure 1C). Vector pLL-EHC, containing centromeric DNA, will represent the centromeric DNA backbone of the $\mathrm{AC}$ construct. We used a rice centromeric BAC 38J12 (Figure 1B) to develop our model pLL-EHC clone. BAC $38 J 12$ contains an $\sim 140-\mathrm{kb}$ insert derived from the centromere of rice chromosome 8 (Cen8) [14]. The insert of this BAC spans the entire $\sim 65-\mathrm{kb}$ CentO centromeric satellite repeat array associated with rice Cen8. An 110kb FseI fragment of the insert, which spans the CentO repeat array, was released from 38J12 and ligated with a FseI-digested pLL-EH vector to generate the pLL-EHC vector (Figure 1). This clone, now considered a centromeric seed clone, will subsequently be combined with telomeric DNA to form AC constructs.

\section{Development of two seed telomeric DNA vectors}

It is well documented that long telomeric repeat arrays can not be stably maintained in E. coli [15]. Our strategy was to clone a short telomeric DNA fragment into seed telomeric DNA vectors. The seed vectors are used as templates to amplify long telomeric DNA fragments, which will then be ligated directly to the centromeric DNA backbone by in vitro site-specific recombination.

A thermostable DNA polymerase from Thermococcus litoralis (Vent DNA polymerase) was used to generate long telomeric repeats from a short synthetic template/ primer following a previously published protocol [16]. We cloned a 340-bp telomeric repeat fragment into the pGEM-T Easy vector. To develop a plasmid that contains a telomeric repeat segment flanked by the appropriate restriction and recombination sites required for future DNA manipulation, the telomeric DNA fragment originally cloned into pGEM-T Easy vector was subcloned into plasmid pTLT (see Materials and Methods). This resulted in two seed telomere vectors: pLL-TBS and pLL-TSB (Figure 2). Propagation of pLL-TBS and pLL-TSB in E. coli strain Top 10 resulted in partial deletions of the 340-bp telomeric DNA fragments. The stabilized pLL-TBS and pLL-TSB plasmids contained only $\sim 120$-bp of the telomeric repeats.

\section{Generation of long "back-to-back" telomeric DNA fragments}

The two seed telomeric DNA vectors contain a homing endonuclease I-SceI site, an attB1 site, and two BsgI sites (Figure 2). The arrangement of the sites differs between the two vectors: BsgI/I-SceI/attB1/MCS/Telo/ BsgI for pLL-TBS, and BsgI/attB1/I-SceI/MCS/Telo/BsgI for pLL-TSB (Figure 2). This arrangement causes the excised long telomeric DNA fragments derived from the two seed vectors to align in opposite orientation such that when later ligated to the ends of the centromeric DNA fragment, produces a linear molecule consisting of a central centromeric DNA element flanked by opposing telomere repeats (Figure 2).

Digesting the pLL-TBS and pLL-TSB vectors with BsgI released the short telomeric DNA inserts, including the attB1 site (Figure 2). The released DNA fragments were used as templates to generate long telomeric DNA fragments by unidirectional replication. This amplification 


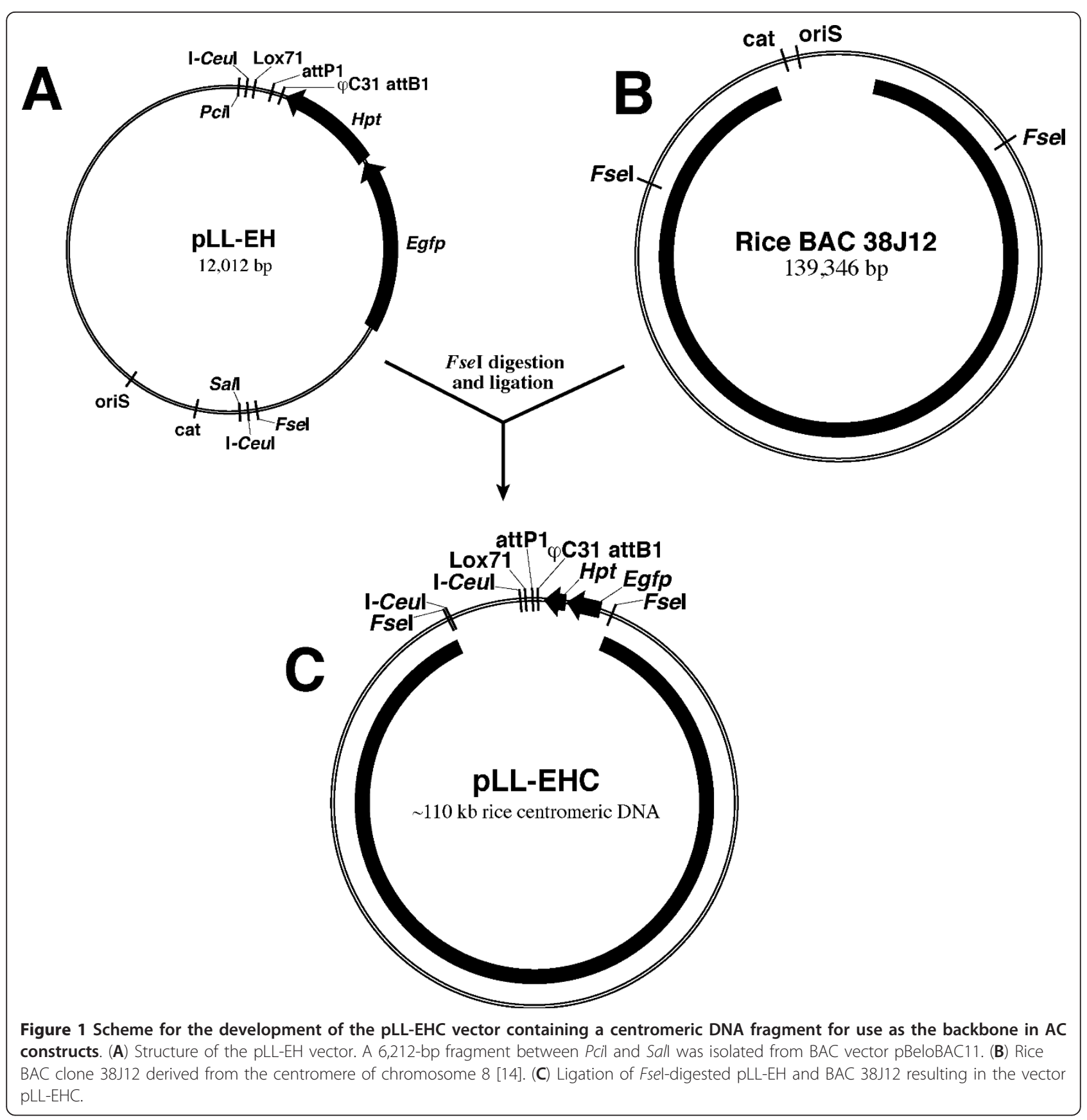

step was accomplished by using Vent DNA polymerase that can catalyze short repeat expansion [16]. DNA fragments in the range of 2-10 kb were readily amplified using this approach (data not shown). The amplified telomeric DNA fragments were size-fractionated via gel excision to generate telomeric DNA varying in lengths (Figure 3A). The 5'-(TTTAGGG) $)_{n}-3^{\prime}$ and 3'(TTTAGGG) (T) $-5^{\prime}$ DNA fragments of 2 to 5 -kb in size were digested with I-SceI and ligated to form back-toback telomeric DNA (Figure 2, Figure 3B). Because the homing endonuclease I-SceI recognizes asymmetric sites and the I-SceI sites on the pLL-TBS and pLL-TSB seed vectors are arranged in an opposite orientation, a telomeric DNA fragment derived from pLL-TBS will only ligate with a fragment derived from pLL-TSB. Thus, the resultant back-to-back telomeric DNA will include two 2 to $5-\mathrm{kb}$ synthetic telomeric DNA fragments in opposite orientation, one I-SceI site, and one attB1 site (Figure 2).

Development and characterization of linear AC constructs The back-to-back telomeric DNA molecules were recombined with the pLL-EHC plasmid containing 


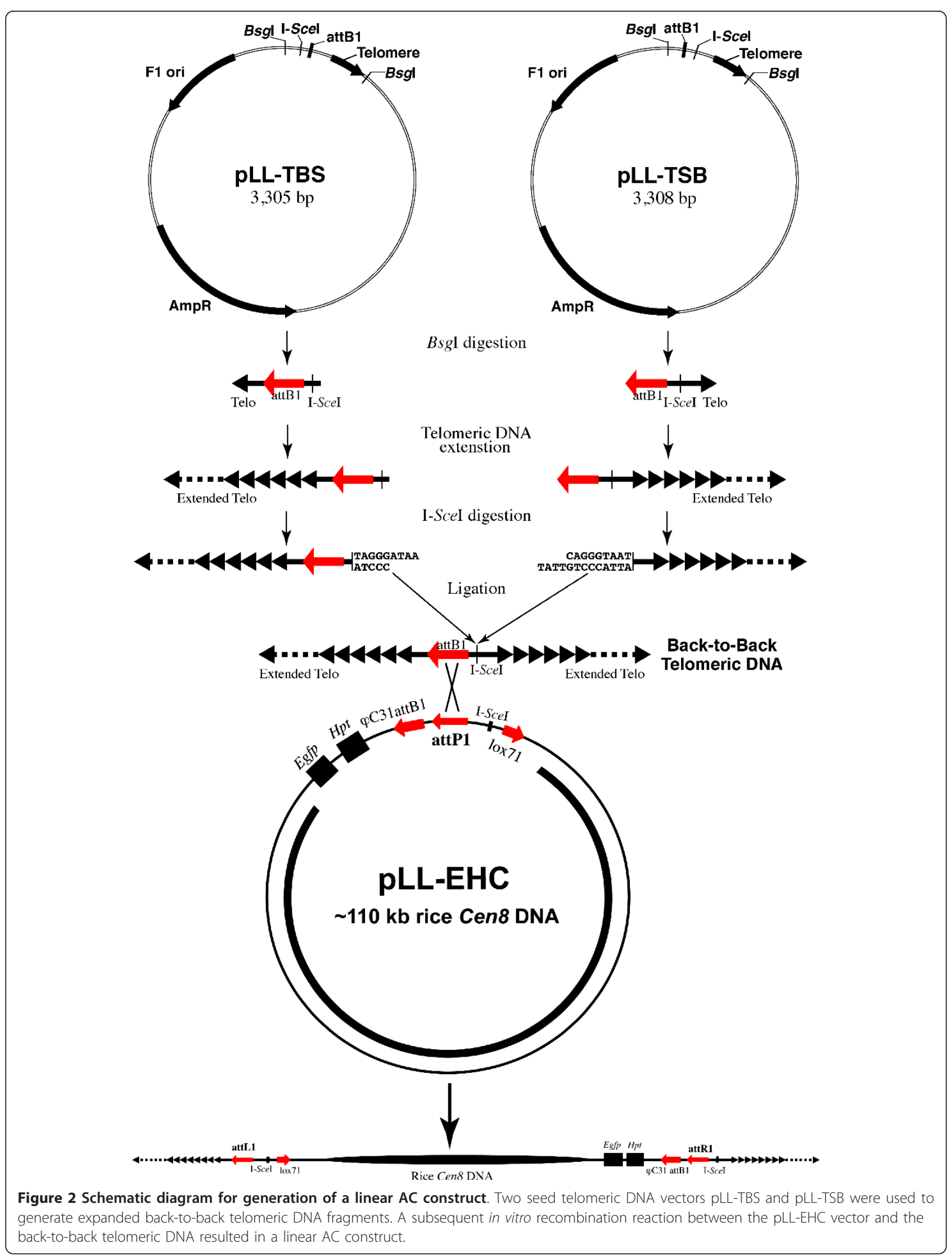




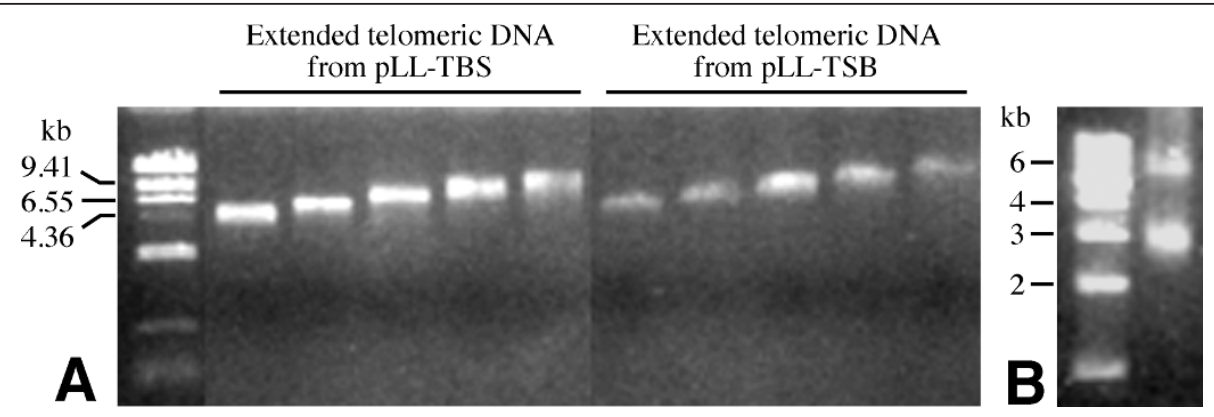

Figure 3 Generation of back-to-back telomeric DNA fragments. (A) Size-fractionated telomeric DNA fragments ranging in size from 4 to 10$\mathrm{kb}$ derived from pLL-TBS and pLL-TSB, respectively. (B) An example of a 6-kb back-to-back telomeric DNA combined from two 3-kb telomeric DNA samples derived from $\mathrm{pLL-TBS}$ and $\mathrm{pLL-TSB}$, respectively. The $3-\mathrm{kb}$ band is the non-recombined telomeric DNA.

centromeric DNA to generate linear AC constructs. The recombination was accomplished through the attB1 site in the telomeric DNA molecule and the attP1 site in the pLL-EHC plasmid (Figure 2). This recombination resulted in a linear molecule consisting of the centromeric DNA fragment derived from pLL-EHC capped with expansive telomeric DNA at both ends. The attP1 and attB1 sites were converted into attL1 and attR1 sites after the recombination (Figure 2). The resulting linear molecules can be used directly for plant transformation with the Hpt gene used as the plant selection marker.

To confirm the recombination between the back-toback telomeric DNA fragment and the pLL-EHC plasmid specific PCR primers were designed from the junction regions based on the backbone sequences of plasmids pLL-EHC, pLL-TBS, and pLL-TSB (Figure 4). The linear AC constructs were isolated by pulsed field gel electrophoresis (PFGE). PCR amplification using the junction-specific primers resulted in DNA fragments matching expected sizes (Figure 4). The amplified PCR fragments were also confirmed by sequencing analysis (data not shown). We also developed linear constructs consisting of centromeric DNA capped with telomeric DNA at only one of the two ends. The single junction associated these constructs was also confirmed by PCR analysis (Figure 4). Southern blot hybridization analysis showed that only AC constructs with telomeric DNA attached at one or both ends hybridized to both telomeric and centromeric DNA probes (Figure 5).

\section{Cytological visualization of linear AC constructs}

We used a DNA fiber-fluorescence in situ hybridization (fiber-FISH) technique to visualize the $\mathrm{AC}$ constructs resulting from the ligations between pLL-EHC and 4 to 8-kb back-to-back telomeric DNA fragments. The recombined DNA was directly spread on poly-lysine coated glass slides and hybridized with telomeric DNA probe (red) and pLL-EHC (green) probes. Linear DNA molecules hybridized with both probes were consistently detected using DNA samples from different ligation experiments (Figure 6). Non-recombined and circular pLL-EHC molecules were also observed. Some linear molecules showed no telomeric DNA signals or a signal at only one of the two ends. However, this is likely due to the resolution limitations of the fiber-FISH technique in which DNA sequences as short as few kilobases are often not detected as consistently as longer DNA fragments.

\section{Discussion}

Methods for generating either de novo artificial chromosomes or engineered minichromosomes can be grouped into two broad categories: the "top-down" and "bottomup" approaches, respectively. The "top-down" approach uses telomeric DNA to truncate a native chromosome thereby generating minichromosomes [17]. Selectable markers can be inserted into such minichromosomes to eventually be engineered into autonomous vectors highly similar to artificial chromosomes [18]. This "topdown" approach has been demonstrated in maize by truncating the supernumerary B chromosome [8]. While truncation of any normal chromosome in a diploid plant species may be lethal, the truncation of a normal chromosome can be achieved in a tetraploid genetic background of the targeted diploid species [8]. Nevertheless, it remains to be seen if such an approach can be applied to a broad set plant species. The "bottom-up" approach assembles AC constructs in vitro using cloned centromeric and telomeric DNA followed by transforming the target plant with the constructs [9].

The centromeric and telomeric DNA compositions of AC constructs can significantly affect the efficiency of artificial chromosome formation [2,19]. Linear centromeric DNA constructs capped with telomeric DNA generated HACs efficiently. However, a severe reduction in HAC formation coupled with an increase in integration events into human chromosomes was observed when the same constructs were not capped with telomeric 

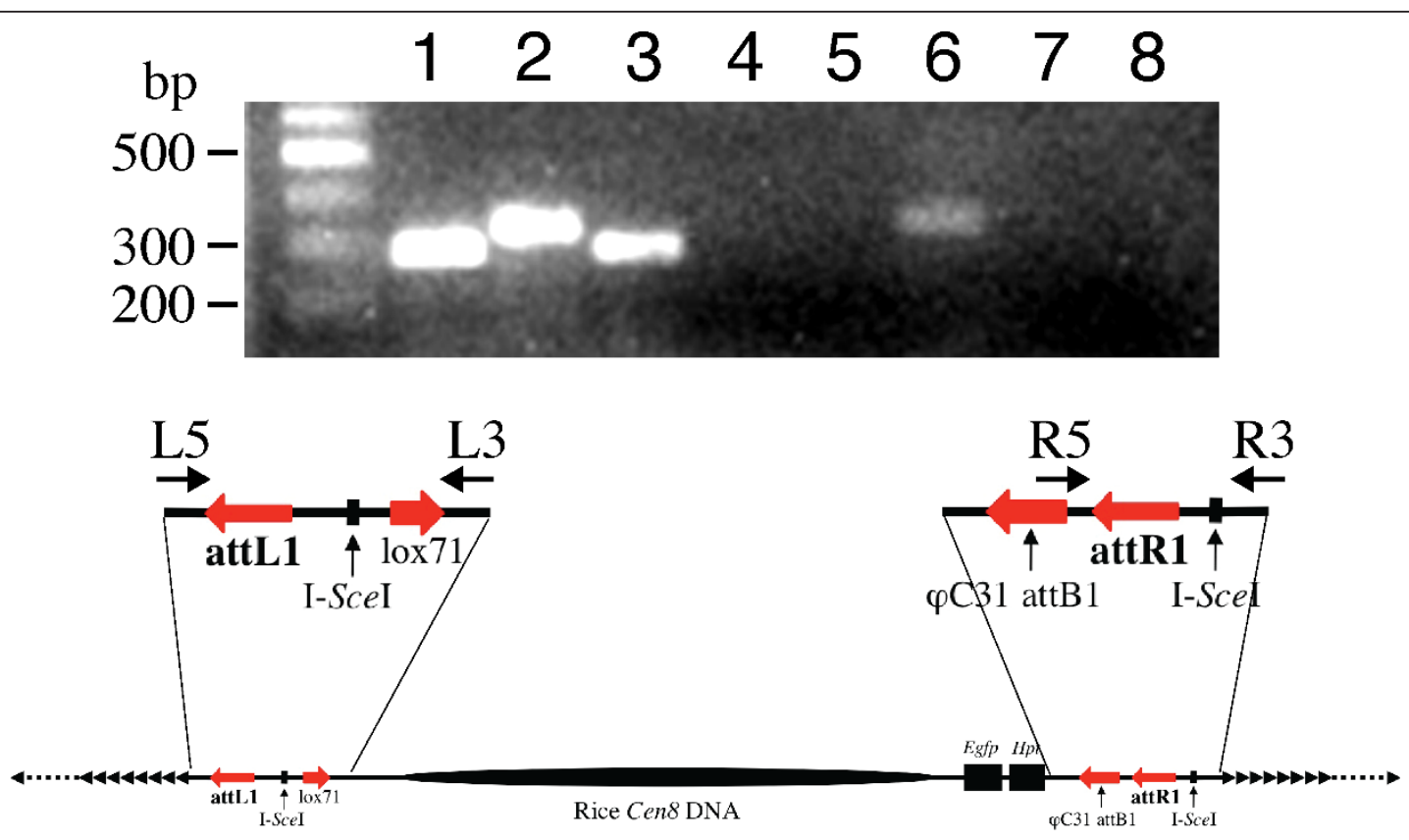

Figure 4 PCR analysis of the linear AC constructs. The primers specific to the left arm junction ( $L 5$ and $L 3)$ and right arm junction (R5 and R3) span the attL1 and attR1 sites, respectively. Lane 1: Left arm junction PCR using linear AC constructs capped with telomeric DNA at both ends. Lane 2: Right arm junction PCR using linear AC constructs capped with telomeric DNA at both ends. Lane 3: Left arm junction PCR using linear AC constructs capped with telomeric DNA at left arm only. Lane 4: Right arm junction PCR using linear AC constructs capped with telomeric DNA at left arm only. Lane 5: Left arm junction PCR using linear AC constructs capped with telomeric DNA at right arm only. Lane 6: Right arm junction PCR using linear AC constructs capped with telomeric DNA at right arm only. Lane 7: Left arm junction PCR using circular pLL-EHC DNA molecules. Lane 8: Right arm junction PCR using circular pLL-EHC DNA molecules.

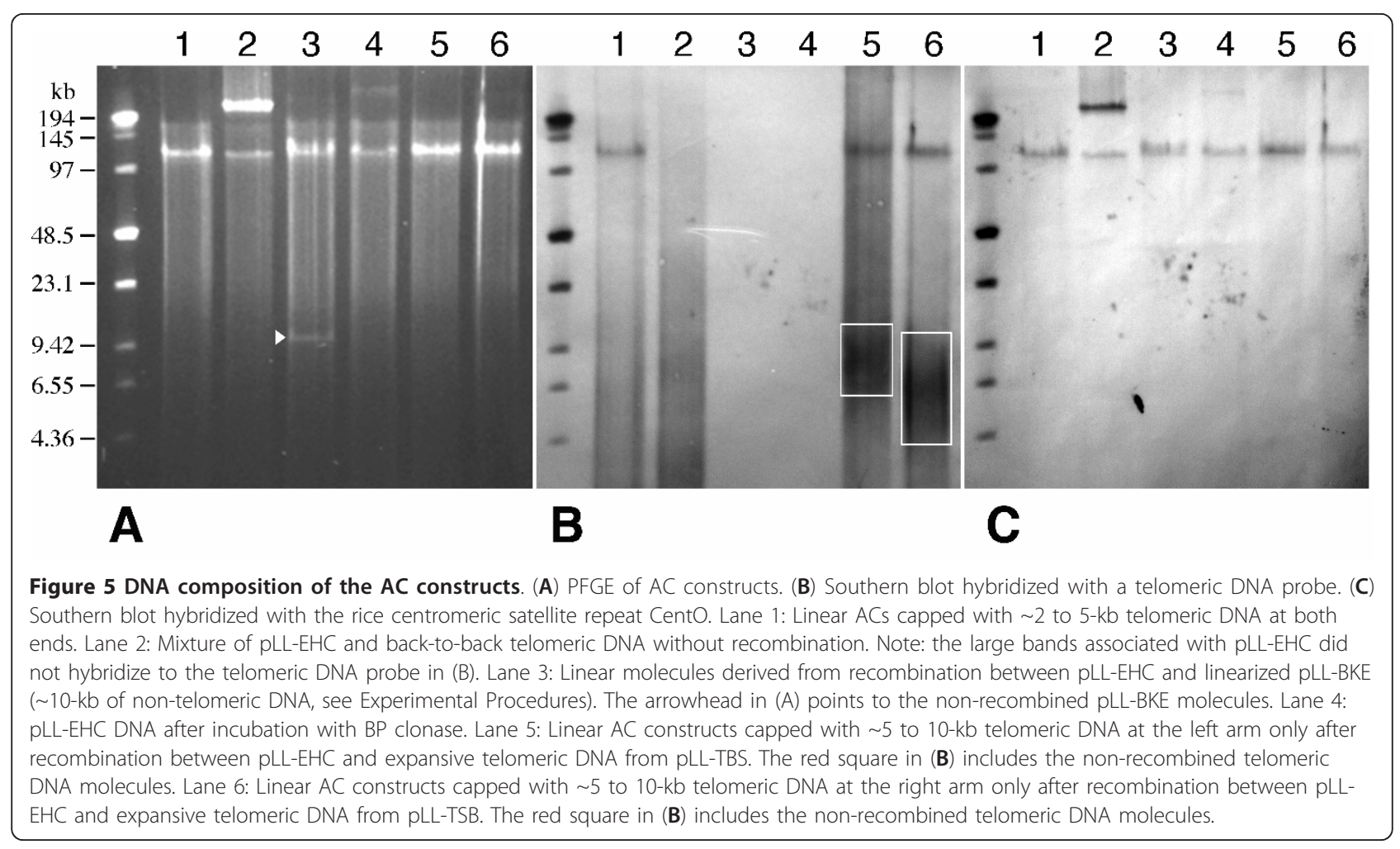


DNA [11]. HACs that acquired long telomeres during in vitro propagation were more stable in mitosis than those with short telomeres [12]. These results indicate that adding long telomeric DNA to linear AC constructs has a major impact on the efficiency of artificial chromosome formation and the stability of the resulting minichromosomes. The telomeric DNA may protect the ends of the linear constructs in a similar function as the telomeres of normal chromosomes. Nevertheless, no specific study has been devoted to finding a relationship between telomeric end size in $\mathrm{AC}$ constructs and the efficiency of artificial chromosome formation.

The average telomere length of human fibroblasts varies from 5 to $10-\mathrm{kb}$ [20]. However, BAC-based HAC constructs were capped by only 0.8 to $1.2-\mathrm{kb}$ of telomeric DNA [19,21-23]. Similarly, only a 239-bp telomeric DNA fragment was included in the BAC-based PAC constructs in maize [9]. It was not specified in these previous reports as to why such short telomeric DNA fragments were included in HAC/PAC constructs. However, it is well known that satellite DNA, such as telomeric repeats [15], are generally not stable in bacterial plasmids, including BAC vectors [24]. Thus, even if BAC-based HAC/PAC constructs containing long telomeric DNA fragments can be developed, it remains unknown if such constructs can be maintained in $E$. coli. It is also interesting to note that putative de novo artificial chromosomes were recovered only 7 of the 450 transformation events using maize AC constructs capped with very short telomeric DNA [9].

It has been well documented that progressive shortening of telomeric DNA can lead to the loss of function of telomeres and the fusion of chromosomes [25]. Thus, it is reasonable to hypothesize that the amount of telomeric sequence capping $\mathrm{AC}$ constructs is important for the efficient formation of artificial chromosomes. We demonstrate that the instability of telomeric repeats can be circumvented by assembling of the AC constructs using expansive telomeric DNA, rather than cloned telomeric DNA. Since most plant species contain the same type of telomeric DNA [(TTTAGGG) $]$ [26], the pLLTBS and pLL-TSB vectors can be used for telomeric DNA amplification and AC construct development in most plant species. In addition, telomeres normally terminate in a 3' single-strand G-rich overhang. This telomeric 3'-overhang is important for the formation of the T-loop, which is believed to protect chromosome ends from being recognized as broken DNA [27]. All previously reported $\mathrm{AC}$ constructs were not capped with telomeric DNA with this 3'-overhange structure due to means by which the telomeric DNA was cloned. Telomeric DNA fragments amplified from pLL-TBS and pLL-TSB do not undergo the cloning process and therefore can be modified to add this 3'-overhang structure before ligating with the centromeric DNA to produce linearized AC constructs.

Although AC constructs capped with sufficient length of telomeric DNA can be generated, PAC research will continue to face the major challenge of delivering such large constructs into plant cells. Currently, microprojectile bombardment is the most popular method to deliver such large constructs. Biolistic transformation of AC constructs will result in chromosomal integration and chromosome truncation events $[9,28]$. It will be interesting to investigate if application of $\mathrm{AC}$ constructs with expansive telomeric DNA will increase or decrase the frequency of such events.

\section{Materials and methods}

\section{Development of backbone plasmids}

The backbone of the pLL-EHC vector was constructed by linking two DNA fragments. The first fragment (6,212-bp) was isolated from the pBeloBAC11 vector [13] by digesting with SalI and PciI (Figure 1A). The second fragment was produced by multiple rounds of oligonucleotide-extension using six different primers. This fragment contains one I-SceI site, two I-CeuI sites, a lox71, an attP1 site, and a multiple cloning site (MCS) consisting of 16 unique restriction sites. The vector pLL-FF was developed by ligating the shared SalI and PciI sites between the synthetic fragment and the pBeloBAC11-derived fragment. A HindIII fragment containing the Egfp gene isolated from the Pk7GWIWG2D(II) vector (Invitrogen, Carlsbad, California) [29] was inserted into pLL-FF to create pLL-E. Finally, a BstXI digested PCR fragment containing the Hpt gene, a plant selectable marker, from pHZWG7 [29], and a synthetic $\phi$ C31 attP1 site were all inserted into PI-PspI digested pLL-E to create pLL-EH (Figure 1). An 110-kb fragment from the rice $\mathrm{BAC}$ OSJNBa0038J12 was then ligated into the FseI site of pLL-EH to yield pLL-EHC.

To generate a telomeric DNA fragment, a PCR reaction was performed using a synthetic (TTTAGGG) 11 DNA fragment as a template and a telomeric 25-mer (TTTAGGG) $)_{3}$ TTTA) as a primer. The reaction was driven using Vent polymerase (1U) (New England Biolabs, Ipswich, Massachusetts) in a buffer containing $20 \mathrm{mM}$ Tris- $\mathrm{HCl}$ (pH 8.8), $10 \mathrm{mM}\left(\mathrm{NH}_{4}\right)_{2} \mathrm{SO}_{4}, 10 \mathrm{mM} \mathrm{KCl}, 2$ $\mathrm{mM} \mathrm{MgSO}_{4}, 0.1 \%$ Triton X-100, 1 mM dNTPs. The concentrations of both telomeric DNA fragments were 1 $\mu \mathrm{M}$ and the volume of the reaction was $5 \mu \mathrm{l}$. The amplified telomeric DNA fragments were cloned into the pGEM-T Easy vector (Promega, Madison, Wisconsin). One recombinant clone containing a 340-bp telomeric DNA fragment, pGEM-TT, was selected and confirmed by direct sequence analysis. The insert of the pGEM-TT plasmid was released using a NdeI and XbaI double digestion, blunt-ended using T4 polymerase (New 
England Biolabs), and subcloned into a PstI-digested and blunt-ended pTLT plasmid. The pTLT plasmid is a modified pGEM-T Easy vector containing two additional BsgI sites. This final clone was named pTLT-R11.

A PCR-based approach was used to insert the I-SceI and attB1 sites into the pTLT-R11 plasmid. The following primers, which contain the attB1 and I-SceI sites, were used in the PCR using pTLT-R11 as a template: TBS5' TTAGTCTCGAGACAAGTTTGTACAAAAAAGCAG GCTCTGCATGCCCTAAATCACTAGTGAATTCG; TBS3' TACTTCTCGAGACAAGTTTGTACAAAAAAGCAGGCTTGGTCTAGACCAAGATATCCTTGGC; TSB5' TTAGTCTCGAGTAGGGATAACAGGGTAATC TGCATGCCCTAAATCACTAGTGAATTCG. TSB3' TACTTCTCGAGTAGGGATAACAGGGTAATTGGTCTAGACCAAGATATCCTTGGC. The PCR fragments were digested with $\mathrm{XhoI}$ and self-ligated to yield the pLLTBS and pLL-TSB plasmids.

\section{Synthesis of back-to-back telomeric DNA fragments}

To generate long telomeric DNA fragments, the short telomeric DNA inserts were released from pLL-TBS and pLL-TSB plasmids by digesting with BsgI. Unidirectional telomeric DNA extension was performed using a 5'(tTTACCC $)_{12}-3$ ' oligonucleotide. The oligonucleotides and the released plasmid inserts were mixed at a 1:2 ratio in a $100 \mu \mathrm{l}$ PCR reaction containing $50 \mathrm{mM}$ Tris$\mathrm{HCl} \mathrm{pH}$ 9.1, $16 \mathrm{mM} \mathrm{NH}_{4} \mathrm{SO}_{4}, 3.5 \mathrm{mM} \mathrm{MgCl}, 150 \mu \mathrm{g} /$ $\mathrm{ml}$ bovine serum albumin (BSA), $250 \mu \mathrm{M}$ dNTPs, Klentaq (5 U) (Clontech, Mountain View, California), and $P f u$ polymerase (0.03 U) (Stratagene, La Jolla, California). The extended DNA fragments were purified and treated with Mung bean nuclease at $30^{\circ} \mathrm{C}$ for 30 min to remove any single stranded DNA. The DNA fragments were then treated with calf intestinal alkaline phosphatase (New England Biolabs) at $37^{\circ} \mathrm{C}$ for $60 \mathrm{~min}$ to remove a phosphate group, ensuring that one DNA fragment from pLL-TBS and one from pLL-TSB would be ligated in a back-to-back direction.

The extended telomeric DNA fragments were separated on $0.7 \%$ low-melting agarose gel. Electrophoresis was performed over night at $37 \mathrm{~V}$. DNA fragments of 2 to $10-\mathrm{kb}$ were excised from the gels. The telomeric DNA was purified from the agarose and concentrated using a Microcon YM-50 spin column (Amicon, Houston, Texas) according to the manufacturer's instructions. Equal amounts of the size-fractionated telomeric DNA derived from pLL-TBS and PLL-TSB were mixed and digested with I-SceI in a total volume of $200 \mu \mathrm{l}$ for $3 \mathrm{~h}$. The homing endonucleases were heat inactivated, and ATP (Epicentre, Madison, Wisconsin) was added to a final concentration of $1 \mathrm{mM}$. The telomeric DNA was ligated overnight at room temperature using T4 DNA ligase.

\section{Assembly of $A C$ constructs}

For the attB1 $\times$ attP1 recombination reaction, $500 \mathrm{ng}$ of the attP1-containg pLL-EHC plasmid DNA and $100 \mathrm{ng}$ of the attB-containing back-to-back telomeric DNA fragments were mixed with $4 \mu \mathrm{l}$ each of $5 \times \mathrm{BP}$ clonase buffer and BP Clonase ${ }^{\mathrm{TM}}$ Enzyme Mix (Invitrogen), and adjusted to $20 \mu \mathrm{l}$ with TE buffer. The mixture was allowed to react at $25^{\circ} \mathrm{C}$ for $16 \mathrm{~h}$. After the recombinationreaction, the enzymes were inactivated by treatment with Proteinase $\mathrm{K}$ for $10 \mathrm{~min}$ at $37^{\circ} \mathrm{C}$. Similar recombination reactions were also performed using the pLLEHC vector and expansive telomeric DNA fragments derived from either pLL-TBS or pLL-TSB alone. This resulted in a polarized capping of the in linear centromeric DNA molecules with telomeric DNA at only one of the two ends.

The assembled linear AC constructs were separated by PFGE. The DNA band corresponding to the expected size of the linear AC constructs was excised from the gel and placed into 0.5 $\times$ TBE. The electro-eluted DNA was then dialyzed into $\mathrm{ddH}_{2} \mathrm{O}$ and concentrated using a Microcon YM-100 spin column (Amicon). The purified DNA fragments were used as a template for PCR analysis (see below). We developed a pLL-BKE plasmid as a control for artificial chromosome confirmation analysis. The pLL-BKE plasmid is a modified pLL-E vector with an attB1 site instead of attP1 site. Recombination between pLL-EHC and a linearized pLL-BKE ( 10 kb), resulted in a linear molecule that is not capped with telomeric DNA.

Specific primers were designed from the junction regions between the pLL-EHC vector and the two telomeric DNA fragments as follow: L5 5'-TGATTTAGGGCATGCAGAGCCTGC-3', L3 5'-CTGTCAAGGGCAAGTATTGACATGT-3', R3 5'-TGATTTAGGGCATGCAGATTA CCC-3', and R5 5'-TCATCTATGTTACTAGAGTACGCGC-3'. The positions of these primers are shown in Figure 6. PCR reaction mixtures contained $1 \mu \mathrm{M}$ primers, $200 \mu \mathrm{M}$ dNTPs, $0.01 \%$ gelatin, $2.5 \mathrm{mM} \mathrm{MgCl}_{2}, 50$ $\mathrm{mM} \mathrm{KCl}, 10 \mathrm{mM}$ Tris- $\mathrm{HCl}$ (pH 8.3), and $1 \mathrm{U}$ rTaq (Takara Bio, Shiga, Japan) in a final volume of $20 \mu$ l. Following an initial denaturing step at $95^{\circ} \mathrm{C}$ for $5 \mathrm{~min}, 35$ amplification cycles of $30 \mathrm{~s}$ at $95^{\circ} \mathrm{C}, 30 \mathrm{~s}$ at $60^{\circ} \mathrm{C}$ and $40 \mathrm{~s}$ at $72^{\circ} \mathrm{C}$, were followed with a final incubation at $72^{\circ} \mathrm{C}$ for $7 \mathrm{~min}$.

\section{Southern blot hybridization and fiber-FISH}

Southern hybridization of DNA was performed using the digoxigenin (DIG) detection system (Boehringer Mannheim BV, Almere, The Netherlands). The probes used in the Southern hybridization were synthesized and DIG-labeled by random priming. The alkali-labile form of DIG-11-dUTP, the pRCS2 plasmid DNA insert containing the CentO repeats [30], and the NotI/NheI digested 140-bp telomere DNA fragments from pTLT- 


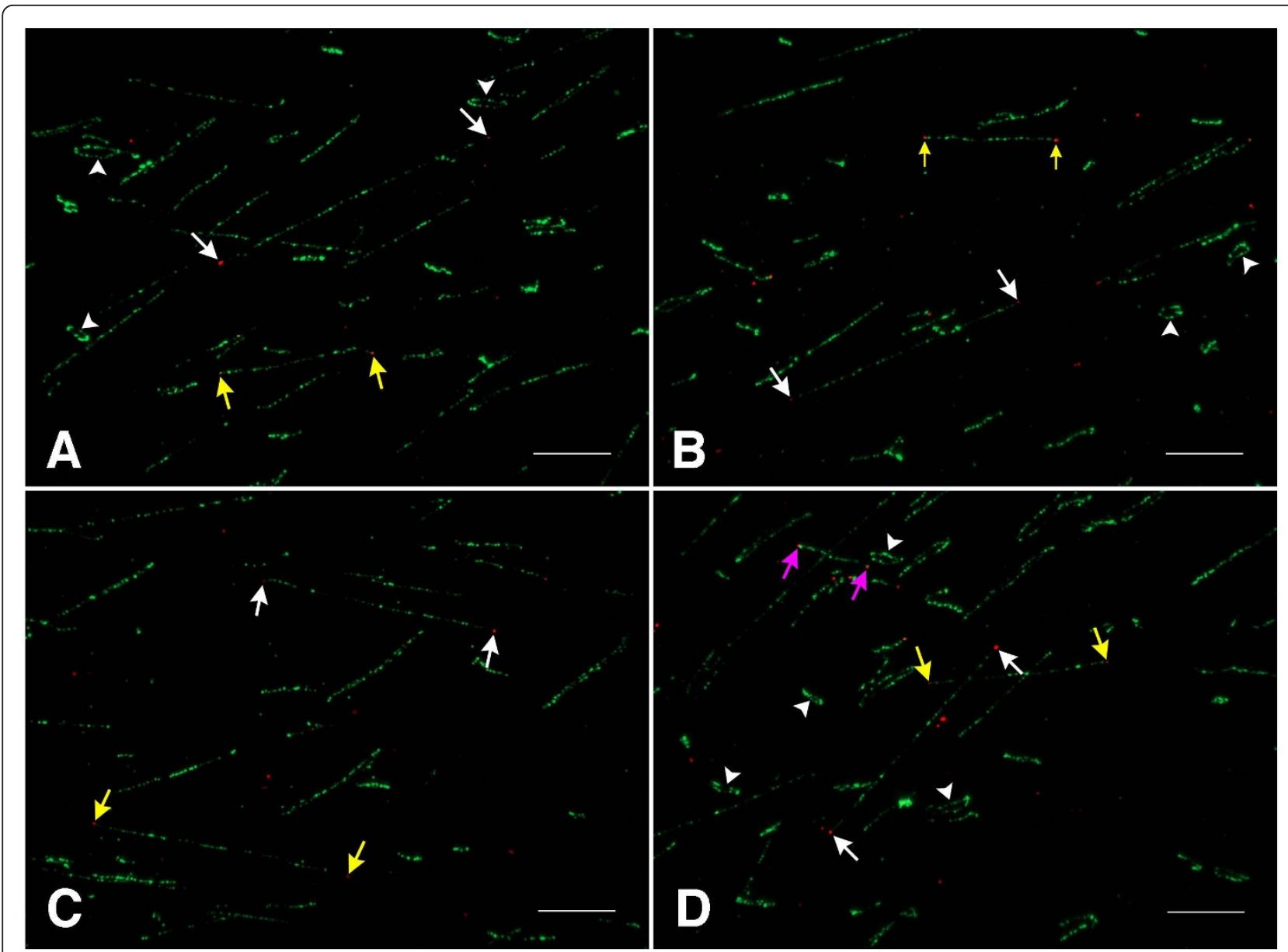

Figure 6 Visualization of linear AC constructs by fiber-FISH using pLL-EHC (green) and telomeric DNA (red), as probes. The two arrows of the same color in the four different images $(\mathbf{A}, \mathbf{B}, \mathbf{C}$, and $\mathbf{D})$ point to the two telomeric signals on the same AC constructs. Arrowheads point to some of the non-recombined circular pLL-EHC molecules. Note: the variability in size of the observed linear and circular DNA molecules is caused by non-uniform extension of the DNA molecules. Bars $=10 \mu \mathrm{m}$.

R11 were used as templates for random labeling with DIG. Hybridization conditions were carried out as recommended by the manufacturer.

Fiber-FISH analysis of assembled AC constructs was performed using published protocols [31]. An appropriate amount of target DNA resulting from ligations between pLL-EHC and 4 to 8-kb of back-to-back oriented telomeric DNA, was directly dropped on a poly-lysine coated glass slide and a $18 \times 18$ cover glass was carefully placed on the top of the DNA drop. Slides were hybridized with a telomeric DNA probe and the pLL-EHC plasmid. The signals were detected following the procedure of standard fiber-FISH [32].

\section{Acknowledgements}

This research was supported by grant DBI-0603927 and DBI-0922703 from the National Science Foundation. We thank Jason Walling and Cory Hirsch for his valuable comments on the manuscript.

\section{Authors' contributions}

LL and JJ conceived and designed the experiments. LL, DHK, WZ, and JSP performed the experiments. $L L$ and $J J$ analyzed the data and wrote the manuscript. All authors read and approved the final manuscript.

\section{Competing interests}

The authors declare that they have no competing interests.

Received: 14 February 2011 Accepted: 15 April 2011 Published: 15 April 2011

\section{References}

1. Murray AW, Szostak JW: Construction of artificial chromosomes in yeast. Nature 1983, 305:189-193.

2. Basu J, Willard HF: Artificial and engineered chromosomes: nonintegrating vectors for gene therapy. Trends Mol Med 2005, 11(5):251-258.

3. Kazuki Y, Hoshiya H, Kai Y, Abe S, Takiguchi M, Osaki M, Kawazoe S, Katoh M, Kanatsu-Shinohara M, Inoue K, Kajitani N, Yoshino T, Shirayoshi Y, Ogura A, Shinohara T, Barrett JC, Oshimura M: Correction of a genetic defect in multipotent germline stem cells using a human artificial chromosome. Gene Ther 2008, 15:617-624.

4. Yu WC, Han FP, Birchler JA: Engineered minichromosornes in plants. Curr Opin Biotech 2007, 18:425-431. 
5. Harrington JJ, Bokkelen GV, Mays RW, Gustashaw K, Willard HF: Formation of de novo centromeres and construction of first-generation human artificial microchromosomes. Nat Genet 1997, 15:345-355.

6. Ikeno M, Grimes B, Okazaki T, Nakano M, Saitoh K, Hoshino H, McGill NI, Cooke H, Masumoto H: Construction of YAC-based mammalian artificial chromosomes. Nat Biotech 1998, 16(5):431-439.

7. Henning KA, Novotny EA, Compton ST, Guan X-Y, Liu PP, Ashlock MA: Human artificial chromosomes generated by modification of a yeast artificial chromosome containing both human alpha satellite and singlecopy DNA sequences. Proc Natl Acad Sci USA 1999, 96:592-597.

8. Yu WC, Han FP, Gao Z, Vega JM, Birchler JA: Construction and behavior of engineered minichromosomes in maize. Proc Nat Acad Sci USA 2007, 104:8924-8929.

9. Ananiev EV, Wu CC, Chamberlin MA, Svitashev S, Schwartz C, GordonKamm W, Tingey S: Artificial chromosome formation in maize (Zea mays L.). Chromosoma 2009, 118:157-177.

10. Costa S, Blow JJ: The elusive determinants of replication origins. EMBO Rep 2007, 8:332-334

11. Ebersole TA, Ross A, Clark E, McGill N, Schindelhauer D, Cooke H, Grimes B: Mammalian artificial chromosome formation from circular alphoid input DNA does not require telomere repeats. Human Mol Genet 2000, 9:1623-1631.

12. Suzuki N, Nishii K, Okazaki T, Ikeno M: Human artificial chromosomes constructed using the bottom-up strategy are stably maintained in mitosis and efficiently transmissible to progeny mice. J Biol Chem 2006, 281:26615-26623.

13. Shizuya H, Birren B, Kim UJ, Mancino V, Slepak T, Tachiiri Y, Simon M: Cloning and stable maintenance of 300-kilobase-pair fragments of human DNA in Escherichia coli using an F-factor-based vector. Proc Nat Acad Sci USA 1992, 89:8794-8797.

14. Nagaki K, Cheng ZK, Ouyang S, Talbert PB, Kim M, Jones KM, Henikoff S, Buell $C R$, Jiang JM: Sequencing of a rice centromere uncovers active genes. Nat Genet 2004, 36:138-145.

15. Yang TJ, Yu YS, Chang SB, de Jong H, Oh CS, Ahn SN, Fang E, Wing RA: Toward closing rice telomere gaps: mapping and sequence characterization of rice subtelomere regions. Theor Appl Genet 2005, 111:467-478

16. Hartig JS, Kool ET: Efficient isothermal expansion of human telomeric and minisatellite repeats by Thermococcus litoralis DNA polymerase. Nucleic Acids Res 2005, 33:4922-4927.

17. Heller R, Brown KE, Burgtorf C, Brown WRA: Mini-chromosomes derived from the human $Y$ chromosome by telomere directed chromosome breakage. Proc Nat Acad Sci USA 1996, 93:7125-7130.

18. Irvine DV, Shaw ML, Choo KHA, Saffery R: Engineering chromosomes for delivery of therapeutic genes. Trends Biotech 2005, 23:575-583.

19. Grimes BR, Rhoades AA, Willard HF: alpha-satellite DNA and vector composition influence rates of human artificial chromosome formation. Mol Ther 2002, 5:798-805.

20. Harley $C B$, Futcher $A B$, Greider $C W$ : Telomeres shorten during aging of human fibroblasts. Nature 1990, 345:458-460.

21. Mejia JE, Larin Z: The assembly of large BACs by in vivo recombination. Genomics 2000, 70:165-170

22. Mejia JE, Alazami A, Willmott A, Marschall P, Levy E, Earnshaw WC, Larin Z: Efficiency of de novo centromere formation in human artificial chromosomes. Genomics 2002, 79:297-304.

23. Basu J, Stromberg G, Compitello G, Willard HF, van Bokkelen G: Rapid creation of BAC-based human artificial chromosome vectors by transposition with synthetic alpha-satellite arrays. Nucleic Acids Res 2005 33:587-596.

24. Song JQ, Dong FG, Lilly JW, Stupar RM, Jiang JM: Instability of bacterial artificial chromosome (BAC) clones containing tandemly repeated DNA sequences. Genome 2001, 44(3):463-469.

25. McKnight TD, Shippen DE: Plant telomere biology. Plant Cell 2004 16:794-803.

26. Fajkus J, Sykorova E, Leitch AR: Telomeres in evolution and evolution of telomeres. Chromosome Res 2005, 13:469-479.

27. de Lange T: Shelterin: the protein complex that shapes and safeguards human telomeres. Genes \& Dev 2005, 19:2100-2110.

28. Phan BH, Jin WW, Topp CN, Zhong CX, Jiang JM, Dawe RK, Parrott WA: Transformation of rice with long DNA-segments consisting of random genomic DNA or centromere-specific DNA. Transgenic Res 2007, 16:341-351.

29. Karimi M, Inze D, Depicker A: GATEWAYTM vectors for Agrobacteriummediated plant transformation. Trends Plant Sci 2002, 7:193-195.

30. Cheng ZK, Dong F, Langdon T, Ouyang S, Buell CB, Gu MH, Blattner FR, Jiang JM: Functional rice centromeres are marked by a satellite repeat and a centromere-specific retrotransposon. Plant Cell 2002, 14:1691-1704.

31. Jackson SA, Dong FG, Jiang JM: Digital mapping of bacterial artificial chromosomes by fluorescence in situ hybridization. Plant J 1999, 17:581-587.

32. Jackson SA, Wang ML, Goodman HM, Jiang JM: Application of fiber-FISH in physical mapping of Arabidopsis thaliana. Genome 1998, 41:566-572.

doi:10.1186/1746-4811-7-10

Cite this article as: Lin et al: De novo assembly of potential linear artificial chromosome constructs capped with expansive telomeric repeats. Plant Methods 2011 7:10.

\section{Submit your next manuscript to BioMed Central and take full advantage of:}

- Convenient online submission

- Thorough peer review

- No space constraints or color figure charges

- Immediate publication on acceptance

- Inclusion in PubMed, CAS, Scopus and Google Scholar

- Research which is freely available for redistribution 